\title{
MULTIPLICITY AND STRUCTURES FOR TRAVELING WAVE SOLUTIONS OF THE KURAMOTO-SIVASHINSKY EQUATION
}

\author{
BAO-FENG FENG
}

Received 6 May 2004

\begin{abstract}
The Kuramoto-Sivashinsky (KS) equation is known as a popular prototype to represent a system in which the transport of energy through nonlinear mode coupling produces a balance between long wavelength instability and short wavelength dissipation. Existing numerical results indicate that the KS equation admits three classes (namely, regular shock, oscillatory shock, and solitary wave) of nonperiodic traveling wave solutions and families of multiple solutions in each class. However, the details of multiple solutions are still unclear because of numerical accuracy. In this work, a rational spectral approach is used to compute these multiple traveling wave solutions. Owing to the high accuracy of the employed method, the new families of regular shock waves are found and the fine structure of each family is recognized.
\end{abstract}

2000 Mathematics Subject Classification: 35A35, 35L05, 35L35, 65M70.

1. Introduction. The Kuramoto-Sivashinsky (KS) equation

$$
u_{t}+u u_{x}+u_{x x}+u_{x x x x}=0
$$

arises as a model amplitude equation in many physical contexts with instability and dissipation. It was originally derived to model phase turbulence in the Belusov-Zhabotinsky reaction [15, 16], and thermal diffusive instability in flame fronts [20]. It has been used to model the long waves on thin films [21], unstable drift waves in plasmas [6, 17]. It has attracted much attention in the past several decades because it serves as a prototype to study spatiotemporal chaos. Extensive numerical experiments of the initial value problem of the KS equation have shown the existence of the periodic cellular patterns $[5,12,13,18]$. These simulations have also addressed the transitions of the dynamical behavior from fixed points (steady solutions) to travelling waves, modulated travelling waves (quasiperiodic solutions), heteroclinic orbits or steady solutions, eventually to "chaos," as the spatial period, that is, the cell length increases. On the other hand, from a theoretical point of view, the existence of an inertial manifold of the KS equation with periodic boundary conditions has been proved [7, 9, 19]. The existence of such a manifold guarantees that the asymptotic behavior of its solutions can be described by a finite-dimensional dynamical system. Low-dimensional behavior of the KS equation is well described by combining the approximate inertial manifold calculations and $\mathrm{O}(2)$ symmetry $[1,2,8,14]$.

On the other hand, the traveling wave solutions of the KS equation has been studied in a finite domain with periodic boundary condition [18] and in an unbounded 
domain [11]. In [11], the authors identified three classes (namely, regular shock, oscillatory shock, and solitary wave) of such solutions and also families of multisolutions in each class. Such solutions are of particular interest as they resemble certain wave disturbances that have been observed in experiments of falling films at low flow rates. Furthermore, it is conjectured that the dynamical behavior of spatiotemporal chaos for the KS equation is relevant to its structures and bifurcation of traveling wave solutions. For example, if all the stationary and periodic solutions are unstable for some parameter range, chaotic behavior of the system is expected, since any orbit governed by the equation has to wander around in the phase space, therefore, the orbits will then lie on strange attractors.

However, the details of multiple solutions are still unclear because of numerical accuracy. In this paper, a mapped Chebyshev pseudospectral method is used to compute the nonperiodic travelling wave solutions of the KS equation (1.1). Owing to the high accuracy of the employed method, the new families of regular shock waves are found and the fine structure of each family is recognized. Furthermore, relations among three classes of solutions are uncovered.

The rest of this paper is organized as follows. In Section 2, the problem is formulated and a far-field analysis is presented to elucidate the possibility of three classes of stationary traveling wave solutions. Then, in Section 3, we present our numerical method and apply it to obtain traveling wave solutions of the KS equation. Finally, in Section 4, some comments and conclusion are given.

2. Preliminary analysis. Because we focus on traveling wave solutions, it is appropriate to carry out a moving coordinate transformation $\partial / \partial t \rightarrow \partial / \partial t-c \partial / \partial x$. This leads to the governing equation

$$
-c u_{x}+u u_{x}+u_{x x}+u_{x x x x}=0 \quad(-\infty<x<\infty)
$$

where $c$ is the wave speed.

Assuming that $u \rightarrow u_{ \pm}$as $x \rightarrow \pm \infty$, where $u_{ \pm}$are constants, (2.1) can be integrated once to yield

$$
-c u+\frac{1}{2} u^{2}+u_{x}+u_{x x x}=-c u_{+}+\frac{1}{2} u_{+}^{2}=-c u_{-}+\frac{1}{2} u_{-}^{2} .
$$

It can be readily deduced that $c=(1 / 2)\left(u_{+}+u_{-}\right)$if $u_{+} \neq u_{-}$, where $c$ is indeterminate at this stage if $u_{+}=u_{-}$. Note that the case $u_{+}=u_{-}=c$ implies the trivial solution $u=u_{+}=u_{-}$. A scale transformation

$$
U=\frac{u-c}{u_{+}-c}, \quad X=\frac{1}{2}\left(u_{+}-c\right) x
$$

converts (2.2) into

$$
\gamma U_{X X X}+U_{X}+U^{2}=1
$$


with $\gamma=(1 / 4)\left(u_{+}-c\right)^{2}$. Equation (2.4) is invariant under transformation $U \rightarrow-U$, $X \rightarrow-X$, which implies that if $U(X)$ is a solution of (2.4), so is $-U(-X)$. Hence, the solutions are either antisymmetric or occur in pairs. It is easily shown that in the threedimensional phase space defined by coordinates $\left(U, U_{X}, U_{X X}\right)$, there exist two critical points, $O_{1}(1,0,0)$ and $O_{2}(-1,0,0)$, for the dynamical system corresponding to (2.4). Assuming $U= \pm 1+a e^{\sigma X}(a \ll 1)$, linearizing (2.4) about $O_{1}$ and $O_{2}$, one obtains the characteristic equations

$$
\sigma^{3}+\sigma \pm 2=0
$$

Roots of the above cubic equation can be written as

$$
\lambda=\mp \lambda_{1}, \pm \frac{1}{2}\left(\lambda_{1}+\mathrm{i} \lambda_{2}\right), \pm \frac{1}{2}\left(\lambda_{1}-\mathrm{i} \lambda_{2}\right),
$$

where

$$
\begin{gathered}
\lambda_{1}=\frac{2}{(3 \gamma)^{1 / 2}} \sinh \theta, \\
\lambda_{2}=\frac{2}{\gamma^{1 / 2}} \cosh \theta, \\
\sinh 3 \theta=3(3 \gamma)^{1 / 2} .
\end{gathered}
$$

As $X \rightarrow \infty$, there is a one-dimensional stable manifold and a two-dimensional unstable focus passing through $O_{1}$, a one-dimensional unstable manifold and a two-dimensional stable focus through $\mathrm{O}_{2}$. As $X \rightarrow-\infty$, the roles of stability or instability are reversed. Traveling wave solutions are classified according to the various ways of connecting a manifold, stable as $X \rightarrow-\infty$, with another manifold which is stable as $X \rightarrow \infty$. Therefore, three types of traveling wave solutions are predicted:

(1) regular shocks: these correspond to the heteroclinic orbits connecting $O_{1}$ and $\mathrm{O}_{2}$. $U$ behaves in the far field as follows:

$$
U \rightarrow \pm 1+a_{ \pm} e^{\mp \lambda_{1} X} \quad(X \rightarrow \pm \infty)
$$

(2) solitary waves: these correspond to the homoclinic orbits of $O_{1}$ or $O_{2}$ (in pairs). For the case $O_{1}$,

$$
\begin{gathered}
U \longrightarrow 1+a e^{-\lambda_{1} X} \quad(X \longrightarrow \infty), \\
U \longrightarrow 1+b e^{(1 / 2) \lambda_{1} X} \cos \left(\frac{1}{2} \lambda_{2} X\right) \quad(X \longrightarrow-\infty),
\end{gathered}
$$

(3) oscillatory shocks: these are also the heteroclinic orbits between $O_{1}$ and $O_{2}$, whereas, the far-field behavior is

$$
U \longrightarrow \mp 1+b_{ \pm} e^{\mp \lambda_{1} X} \cos \left(\frac{1}{2} \lambda_{2} X\right) \quad(X \longrightarrow \pm \infty) .
$$


Before proceeding to the next section, we carry out a transformation $X \rightarrow \lambda_{1} X$, which leads (2.4) to

$$
v U_{X X X}+(1-v) U_{X}=\frac{1}{2}\left(1-U^{2}\right)
$$

where $v=1-(1 / 2) \lambda_{1}$. Equation (2.10) is used in our following numerical computation.

3. Numerical method and results. In this section, we will present an effective numerical method for computing solitary wave solutions, which is called the rational Chebyshev pseudospectral method by Boyd [3]. The earliest idea was by Grosch and Orszag [10] and was given a complete treatment by Boyd [4].

3.1. Mapped Chebyshev pseudospectral method. The basis functions we used are mapped Chebyshev polynomials:

$$
T B_{n}(X)=\cos \left(n \cot ^{-1}\left(\frac{X}{L}\right)\right), \quad n=0,1, \ldots
$$

where $L$ is the mapped parameter. With the map $t=X / \sqrt{L^{2}+X^{2}}$, our basis functions, as defined in (3.1), are equal to $T_{n}(t)$, where $T_{n}(t)$ are the usual Chebyshev polynomials. The first five basis functions with $L=1$ are

$$
\begin{gathered}
T B_{0}(X)=1, \quad T B_{1}(X)=\frac{X}{\sqrt{X^{2}+1}}, \quad T B_{2}(X)=\frac{X^{2}-1}{X^{2}+1}, \\
T B_{3}(X)=\frac{X\left(X^{2}-3\right)}{\left(X^{2}+1\right)^{3 / 2}}, \quad T B_{4}(X)=\frac{X^{4}-6 X^{2}+1}{\left(X^{2}+1\right)^{2}} .
\end{gathered}
$$

The rate of convergence of the $T B_{n}(X)$ series is normally exponential but subgeometric. The orthogonality relation with $L=1$ is

$$
\int_{-\infty}^{\infty} \frac{T B_{m}(X) T B_{n}(X)}{1+X^{2}} d X=\frac{\pi d_{n}}{2} \delta_{m, n}
$$

where $d_{0}=2, d_{n}=1(n \geq 1)$, and $\delta_{m, n}$ is the Kronecker delta.

Since we are to solve (2.10) in an unbounded domain $X \in(-\infty, \infty)$, it is convenient to expand the solution as

$$
U(X)=\sum_{n=1}^{N} r_{j} \phi_{j}(X),
$$

where $N$ is a given positive integer. $\phi_{j}(X), j=1,2, \ldots, N$, are taken to be

$$
\phi_{j}(X)= \begin{cases}T B_{2 j-1}(X) & \text { for shock waves, } \\ T B_{j-1}(X) & \text { for solitary waves }\end{cases}
$$


which implies that the solutions are antisymmetric for shock waves. The corresponding collocation points $X_{i}, i=1,2, \ldots, N$ are chosen as

$$
X_{i}= \begin{cases}\cot \left(\frac{2 i-1}{4 N} \pi\right) & \text { for shock waves } \\ \cot \left(\frac{2 i-1}{2 N} \pi\right) & \text { for solitary waves }\end{cases}
$$

Furthermore, we should have the boundary condition

$$
\left.U(X)\right|_{X=\infty}=\sum_{j=1}^{N} r_{j}=U_{\infty}
$$

where $U_{\infty}=1$ for regular shock and solitary waves, and $U_{\infty}=-1$ for oscillatory shock waves. For the pseudospectral method, we have (2.10) to be satisfied at the collocation points $X_{i}, i=1,2, \ldots, N$. This result, together with (3.7), yields $N+1$ equations for the $N+1$ unknowns $r_{j}, j=1,2, \ldots, N$, and $v$.

The above $N+1$ nonlinear equations can be solved with the Newton-Kantorovich iteration method. Firstly, we give a guess $U^{(0)}(X)$ and $v^{(0)}$, in the $i$ th iterate, we suppose

$$
U^{(i+1)}(X)=U^{(i)}(X)+\Delta(X), \quad v^{(i+1)}=v^{(i)}+\Delta v .
$$

By substituting (3.8) into the $N+1$ nonlinear equations and ignoring the higher-order small terms, we arrive at $N+1$ linear equations, which can be solved by Gaussian elimination.

\subsection{Numerical results}

3.2.1. Regular shocks. The regular shock wave solutions are antisymmetric, which correspond to the heteroclinic orbits connecting the two fixed points of (2.4). Two new families of solutions are found in our numerical computation. The first five members of the first family are depicted in Figure 3.1 with $\gamma=0.36083977,0.34877725,0.34930413$, $0.34928172,0.34928266$. It is worth noting that approximately, we have $\left(\gamma_{i+1}-\gamma_{i}\right) /$ $\left(\gamma_{i+2}-\gamma_{i+1}\right) \approx-23$. Although we only determine the first five members of this family, we conjecture that there is a countable number of members with $\gamma_{1}, \gamma_{2}, \ldots, \gamma_{n} \rightarrow \gamma_{c}$ between $(0.34877725,0.36083977)$. The solution corresponding to $\gamma_{n}$ has $n$ "humps" in the domain $X \in(-\infty, \infty)$. We suppose that $\gamma_{c}$ is very close to 0.34928266 .

The first five members of the second family are shown in Figure 3.2 with $\gamma=$ $0.12394728,0.11739917,0.11839120,0.11824900,0.11826914$. Again, they have the relation approximately $\left(\gamma_{i+1}-\gamma_{i} / \gamma_{i+2}-\gamma_{i+1}\right) \approx-7$. We conjecture that there is also a countable number of members in this family between the first two eigenvalues $\gamma=$ 0.12394728 and $\gamma=0.11739917$. Their eigenvalues approach a limit value, which is estimated just less than 0.11826914 .

3.2.2. Solitary waves. The solitary wave solutions correspond to the homoclinic orbits around either of the two fixed points of (2.4). Two families of such solutions are obtained numerically. 


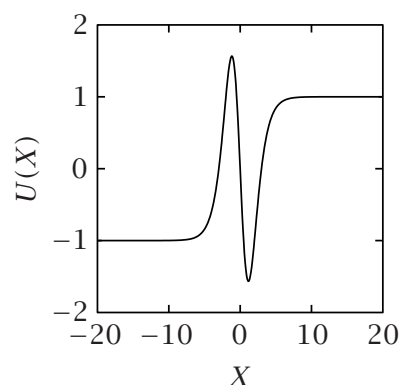

(a)

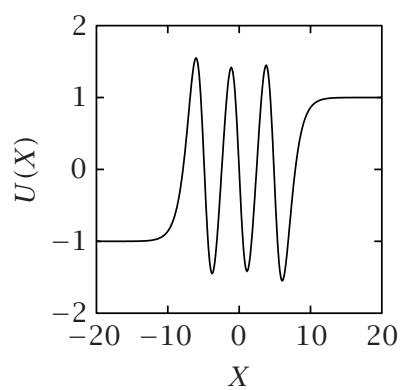

(c)

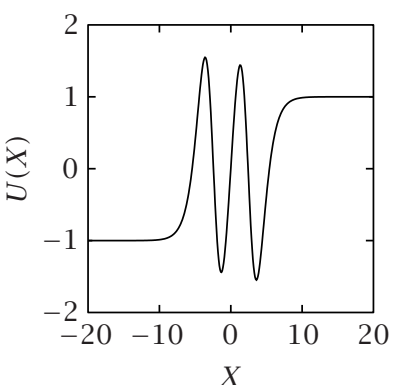

(b)

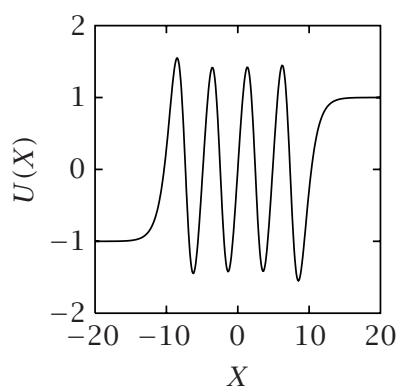

(d)

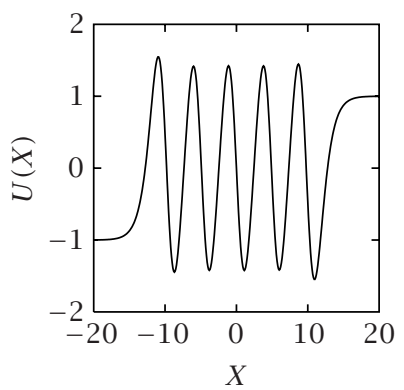

(e)

FIGURE 3.1. Numerical solutions for the first family of regular shocks: (a) $\gamma=$ 0.36083977 , (b) $\gamma=0.34877725$, (c) $\gamma=0.34930413$, (d) $\gamma=0.34928172$, (e) $\gamma=0.34928266$.

The first five members of the first family are shown in Figure 3.3 with $\gamma=0.36975528$, $0.34837892,0.34932100,0.34928100,0.34928271$. It is clear that they are corresponding to the first family of regular shock waves. For the same number "humps," the eigenvalue of each member is very close to that of the member in the corresponding regular 


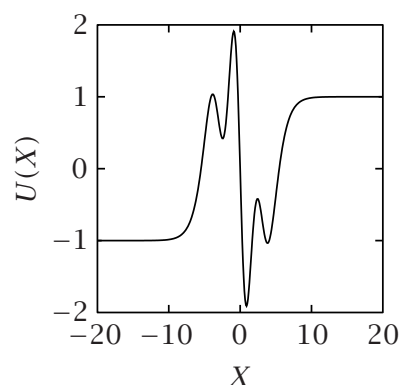

(a)

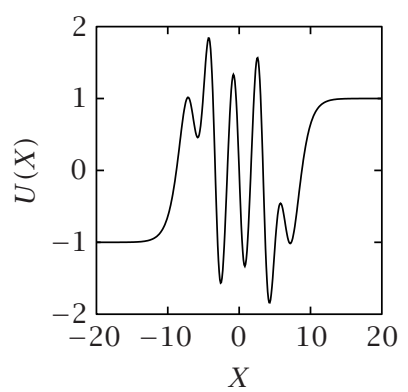

(c)

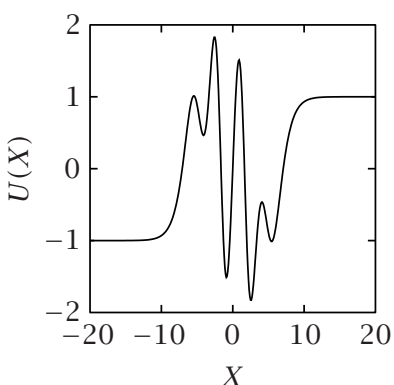

(b)

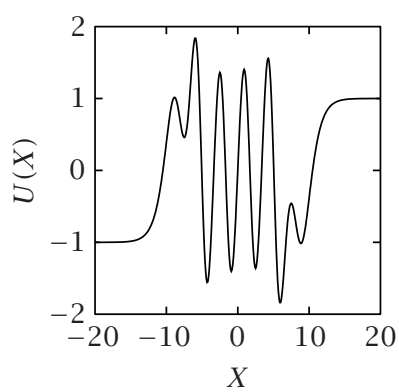

(d)

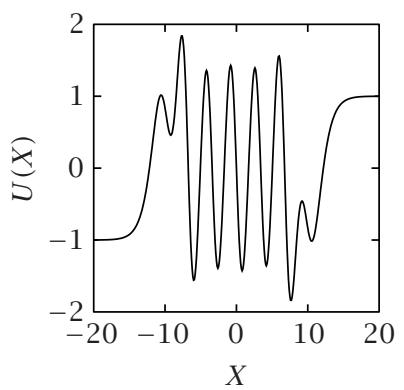

(e)

FIGURE 3.2. Numerical solutions for the second family of regular shocks: (a) $\gamma=0.12394728$, (b) $\gamma=0.11739917$, (c) $\gamma=0.11839120$, (d) $\gamma=0.11824900$, (e) $\gamma=0.11826914$.

shocks family. We conjecture here that there is a countable number of members in this family. Their eigenvalues approach a limit value $\gamma_{e}$, which should be close to $\gamma_{c}$ up to any decimal number, but different from $\gamma_{c}$. It is impossible to distinguish between them numerically. 


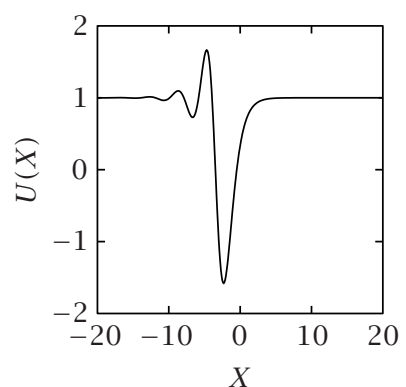

(a)

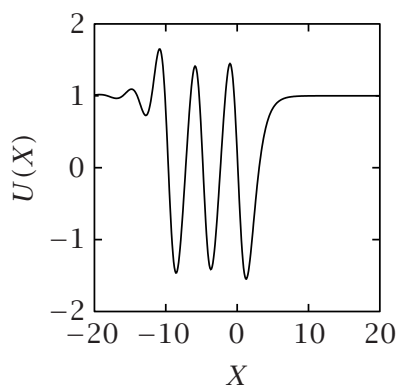

(c)

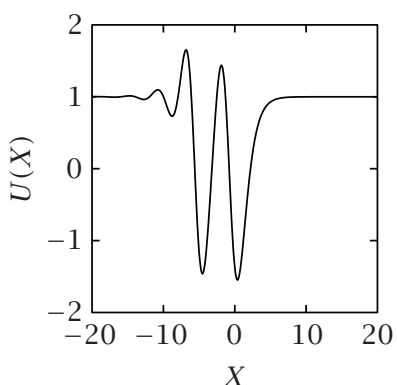

(b)

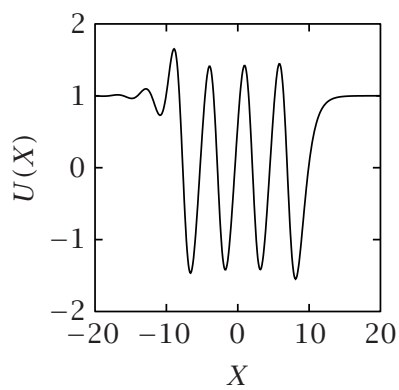

(d)

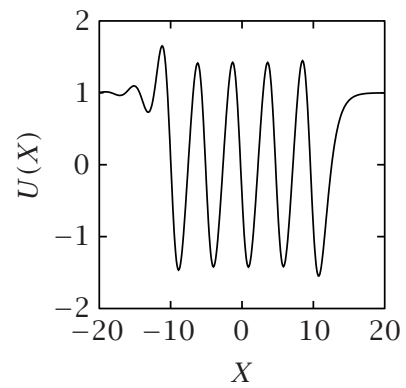

(e)

FIGURE 3.3. Numerical solutions for the first family of solitary waves: (a) $\gamma=$ 0.36975528 , (b) $\gamma=0.34837892$, (c) $\gamma=0.34932100$, (d) $\gamma=0.34928100$, (e) $\gamma=0.34928271$.

Similarly, the first five members of the second family are depicted in Figure 3.4 with $\gamma=0.12410820,0.11737594,0.11839452,0.11824886,0.11826924$. Again, the above conjecture is confirmed by our numerical results. 


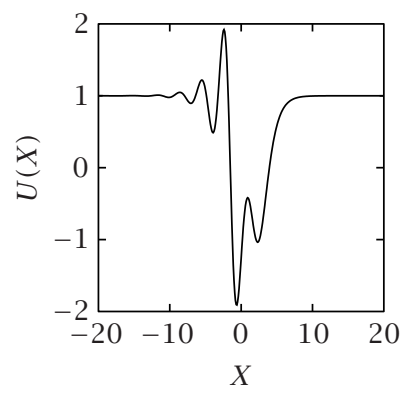

(a)

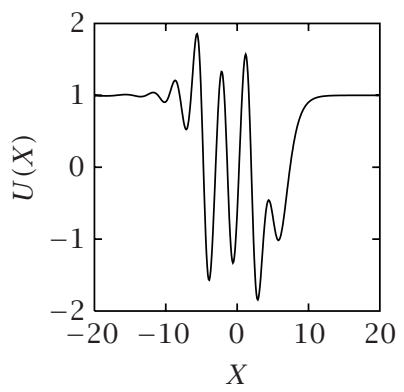

(c)

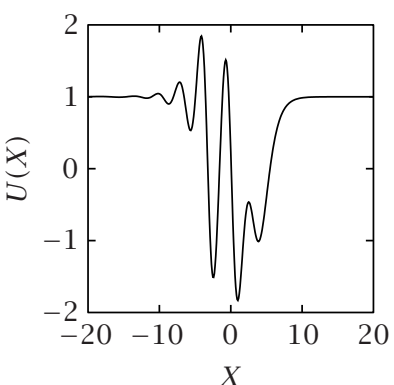

(b)

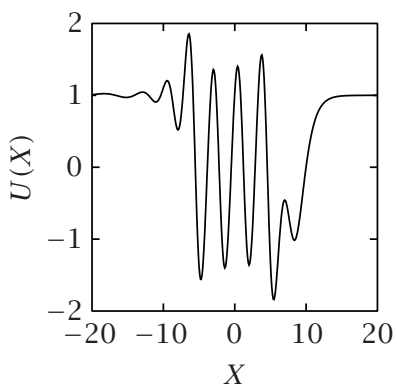

(d)

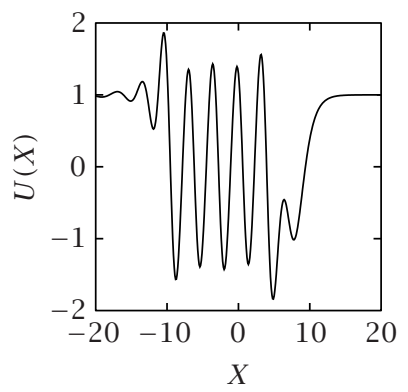

(e)

FIGURE 3.4. Numerical solutions for the second family of solitary waves: (a) $\gamma=0.12410820$, (b) $\gamma=0.11737594$, (c) $\gamma=0.11839452$, (d) $\gamma=0.11824886$, (e) $\gamma=0.11826924$.

4. Comments and conclusion. In the present paper, a rational spectral method is developed for solving nonlinear boundary problems with an application of finding the stationary traveling wave solutions of Kuramoto-Sivashinsky equation. Because the basis functions used are mapped Chebyshev polynomials, this method does not require the infinite domain to be truncated. The numerical results indicate that the rational spectral method can produce very accurate numerical solutions. 
With the help of the new method, the new families of regular shock waves are found and the fine structure of each family is recognized. Furthermore, relations among three classes of solutions are uncovered.

\section{REFERENCES}

[1] D. Armbruster, J. Guckenheimer, and P. Holmes, Heteroclinic cycles and modulated travelling waves in systems with O(2) symmetry, Phys. D 29 (1988), no. 3, 257-282.

[2] _ Kuramoto-Sivashinsky dynamics on the center-unstable manifold, SIAM J. Appl. Math. 49 (1989), no. 3, 676-691.

[3] J. P. Boyd, Spectral methods using rational basis functions on an infinite interval, J. Comput. Phys. 69 (1987), no. 1, 112-142.

[4]_Chebyshev and Fourier Spectral Methods, Springer-Verlag, New York, 1989.

[5] H.-C. Chang, Traveling waves on fluid interfaces: normal form analysis of the KuramotoSivashinsky equation, Phys. Fluids 29 (1986), no. 10, 3142-3147.

[6] B. I. Cohen, J. A. Krommes, W. M. Tang, and M. N. Rosenbluth, Nonlinear saturation of the dissipative trapped-ion mode by mode coupling, Nuclear Fusion 16 (1976), 971-992.

[7] P. Collet, J.-P. Eckmann, H. Epstein, and J. Stubbe, A global attracting set for the KuramotoSivashinsky equation, Comm. Math. Phys. 152 (1993), no. 1, 203-214.

[8] S. P. Dawson and A. M. Mancho, Collections of heteroclinic cycles in the KuramotoSivashinsky equation, Phys. D 100 (1997), no. 3-4, 231-256.

[9] J. Goodman, Stability of the Kuramoto-Sivashinsky and related systems, Comm. Pure Appl. Math. 47 (1994), no. 3, 293-306.

[10] C. E. Grosch and S. A. Orszag, Numerical solution of problems in unbounded regions: coordinate transforms, J. Comput. Phys. 25 (1977), no. 3, 273-295.

[11] A. P. Hooper and R. Grimshaw, Travelling wave solutions of the Kuramoto-Sivashinsky equation, Wave Motion 10 (1988), no. 5, 405-420.

[12] J. M. Hyman and B. Nicolaenko, The Kuramoto-Sivashinsky equation: a bridge between PDEs and dynamical systems, Phys. D 18 (1986), no. 1-3, 113-126.

[13] J. M. Hyman, B. Nicolaenko, and S. Zaleski, Order and complexity in the KuramotoSivashinsky model of weakly turbulent interfaces, Phys. D 23 (1986), no. 1-3, 265292.

[14] I. G. Kevrekidis, B. Nicolaenko, and J. C. Scovel, Back in the saddle again: a computer assisted study of the Kuramoto-Sivashinsky equation, SIAM J. Appl. Math. 50 (1990), no. 3, 760-790.

[15] Y. Kuramoto, Chemical Oscillations, Waves, and Turbulence, Springer Series in Synergetics, vol. 19, Springer-Verlag, Berlin, 1984.

[16] Y. Kuramoto and T. Tsuzuki, Persistent propagation of concentration waves in dissipative media far from thermal equilibrium, Progr. Theoret. Phys. 55 (1976), 356-369.

[17] R. E. LaQuey, S. M. Mahajan, P. H. Rutherford, and W. M. Tang, Nonlinear saturation of the trapped-ion mode, Phys. Rev. Lett. 34 (1975), 391-394.

[18] D. Michelson, Steady solutions of the Kuramoto-Sivashinsky equation, Phys. D 19 (1986), no. 1, 89-111.

[19] B. Nicolaenko, B. Scheurer, and R. Temam, Some global dynamical properties of the Kuramoto-Sivashinsky equations: nonlinear stability and attractors, Phys. D 16 (1985), no. 2, 155-183.

[20] G. I. Sivashinsky, Nonlinear analysis of hydrodynamic instability in laminar flames. I. Derivation of basic equations, Acta Astronaut. 4 (1977), no. 11-12, 1177-1206.

[21] J. Topper and T. Kawahara, Approximate equations for long nonlinear waves on a viscous fluid, J. Phys. Soc. Japan 44 (1978), no. 2, 663-666.

Bao-Feng Feng: Department of Mathematics, The University of Texas - Pan American, Edinburg, TX 78541-2999, USA

E-mail address: feng@panam.edu 


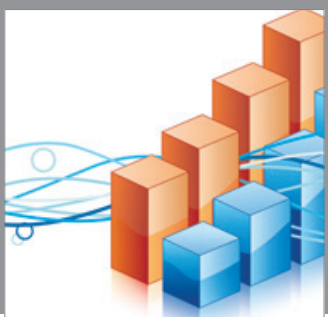

Advances in

Operations Research

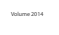

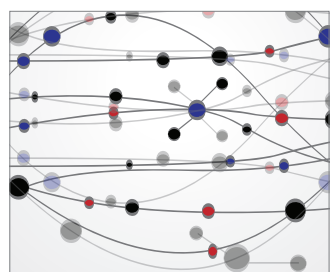

\section{The Scientific} World Journal
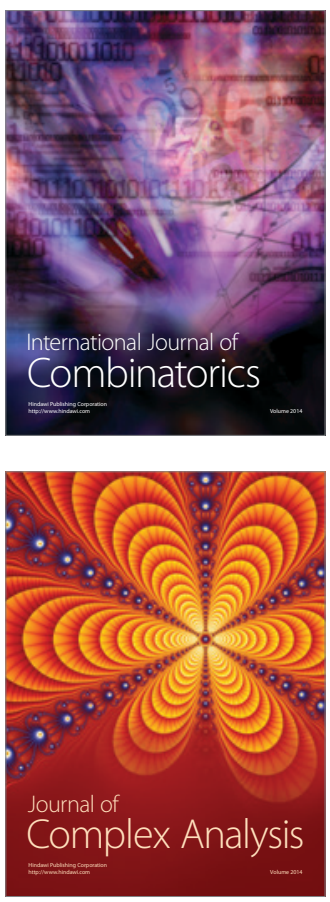

International Journal of

Mathematics and

Mathematical

Sciences
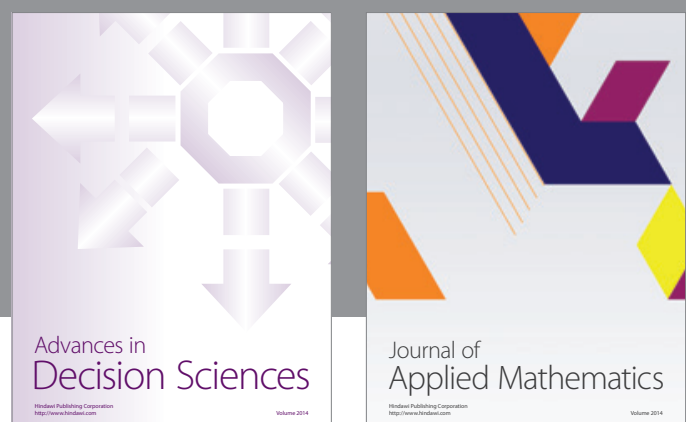

Journal of

Applied Mathematics
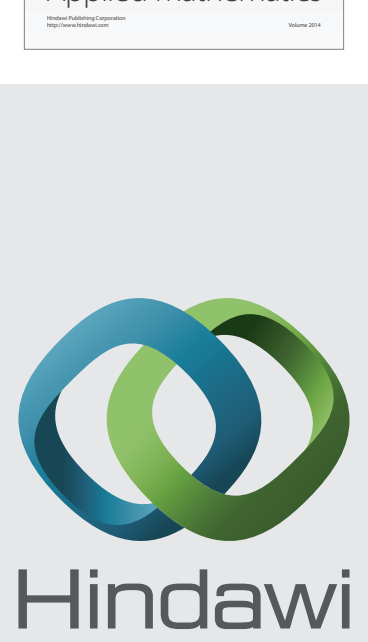

Submit your manuscripts at http://www.hindawi.com
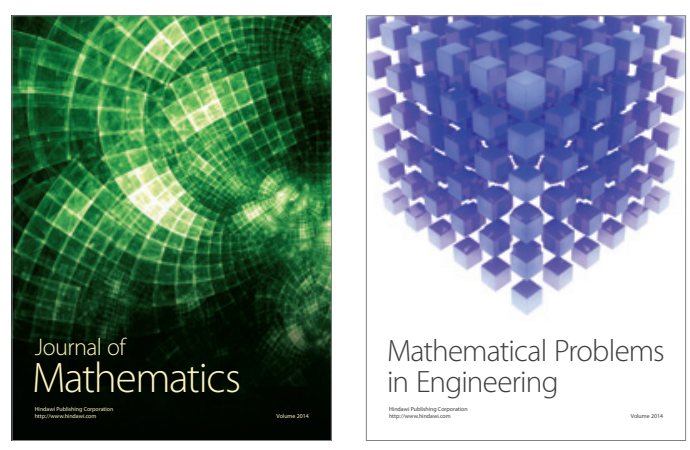

Mathematical Problems in Engineering
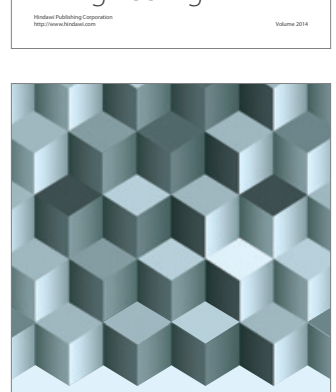

Journal of

Function Spaces
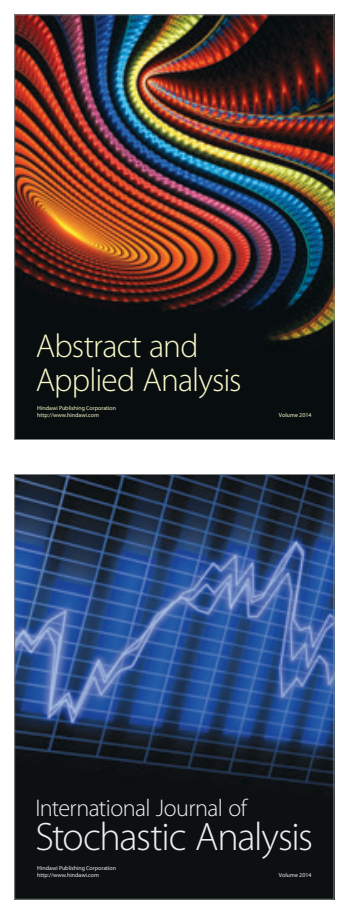

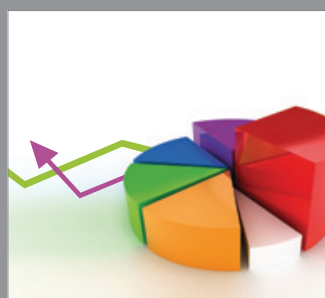

ournal of

Probability and Statistics

Promensencen
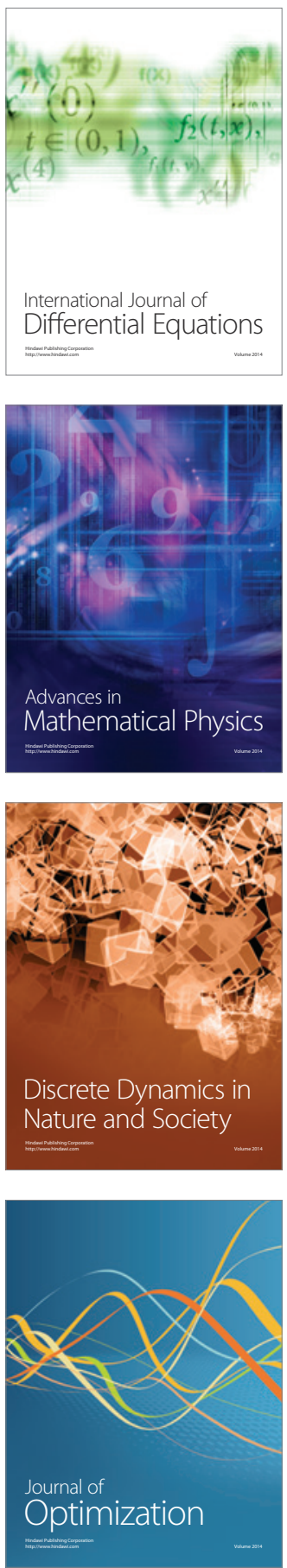